\title{
PRÁCTICAS DE CUIDADO CULTURAL EN EL CONTINUO REPRODUCTIVO DE LA MUJER EMBERA KATIO DEL ALTO SINÚ.
}

\section{CULTURAL CARE PRACTICES IN THE REPRODUCTIVE CONTINUUM OF EMBERA KATIO WOMEN OF THE ALTO SINÚ.}

\author{
PRÁCTICAS DE ATENDIMENTO CULTURAL NO CONTINUO \\ REPRODUTIVO DE MULHERES EMBERA KATIO DEL ALTO SINÚ.
}

\author{
Javier A Bula Romero \\ Universidad de Córdoba, Colombia. \\ javierbula@correo.unicordoba.edu.co \\ ORCID: 0000-0002-0788-0472 \\ Luz Enith Maza Padilla \\ Universidad de Córdoba, Colombia. \\ ORCID: 0000-0002-6289-3321 \\ Martha Orozco Valeta \\ Universidad de Córdoba, Colombia. \\ ORCID: 0000-0002-6217-0908
}

DOI: https://doi.org/10.22235/ech.v8i1.1785
Recibido: $12 / 10 / 2018$
Aceptado: $05 / 03 / 2018$

\section{RESUMEN:}

El cuidado está presente en todas las culturas y tiene características diferentes. Una etapa del curso de vida donde se evidencia con más claridad el cuidado cultural en las comunidades indígenas es en la etapa reproductiva. Este trabajo se trata acerca de una investigación cualitativa de tipo etnográfico interpretativo, que busca describir, explicar e interpretar las prácticas de cuidado cultural en el continuo reproductivo de la mujer Embera Katio. Emergieron cuatro categorías de análisis: preparación de la mujer Embera para albergar la semilla que brotará de sus entrañas; resguardando la semilla del pueblo Embera en el vientre materno; cuando brota la semilla: surge una nueva vida y, la armonía y restablecimiento de la salud en la diada madre-hijo. Se concluye que el continuo reproductivo de la mujer Embera Katio está representado por un conjunto de prácticas de cuidado de orden intergeneracional, que se encuentran soportadas en un conocimiento y comportamiento cultural específico, que garantiza la protección de la mujer y su descendencia. Se destacan en esta síntesis interpretativa las siguientes prácticas de cuidado cultural: el Jemené: rito que marca el inicio de la mujer Embera Katio a la vida fértil; la alimentación: una manera de preservar el bienestar de la madre y su hijo por nacer; la verticalización del parto y el uso de plantas medicinales durante el trabajo de parto; los consejos y el acompañamiento de la partera tradicional durante el embarazo, parto y posparto y por último, el reposo y la armonía de la madre y el recién nacido en el posparto.

Palabras clave: Mujer Indígena, Grupos Étnicos, Prácticas, Cuidado Cultural. 


\begin{abstract}
:
Care is present in all cultures and has different characteristics in each one of them; a stage of the life course where the cultural care in the indigenous communities is most clearly evidente is in the reproductive stage. Qualitative ethnographic interpretive research, which seeks to describe, explain and interpret cultural care practices in the reproductive continuum of Embera Katio women. Results: four categories of analysis emerged as described below: preparation of the Embera woman to house the seed that will sprout from her; sheltering the seed of the Embera people in the mother's womb; when the seed sprouts: a new life emerges and the harmony and restoration of health in the mother-child dyad. Conclusions: the reproductive continuum of the Embera Katio woman is represented by a set of care practices of intergenerational order, which are supported by knowledge and specific cultural behavior, which guarantees the protection of the woman and her offspring. The following cultural care practices stand out in this interpretive synthesis: the Jemené: rite that marks the beginning of the Embera Katio woman to the fertile life; feeding: a way to preserve the welfare of the mother and her unborn child; the verticalization of childbirth and the use of medicinal plants during labor; the advice and accompaniment of the traditional midwife during pregnancy, childbirth and postpartum and, lastly, rest and harmony of the mother and newborn in the postpartum period.
\end{abstract}

Keywords: Indigenous Women, Ethnic Groups, Practices, Cultural Care.

\title{
RESUMO:
}

O cuidado está presente em todas as culturas e possui características diferentes em cada uma delas. Uma etapa do curso de vida em que o cuidado cultural nas comunidades indígenas é mais evidente é no estágio reprodutivo. Este trabalho trata de uma pesquisa qualitativa de tipo etnográfico interpretativo, a qual procura descrever, explicar e interpretar práticas de cuidado cultural no continuum reprodutivo de mulheres de Embera Katio. Surgiram quatro categorias de análise, conforme descrito a seguir: preparação da mulher Embera para abrigar a semente que brotará de dentro dela; abrigando a semente do povo Embera no ventre da mãe. Quando a semente brota, emerge tanto uma nova vida quanto a harmonia e restauração da saúde na díade mãe-filho. Concluise que o continuum reprodutivo das mulheres de Embera Katio é representado por um conjunto de práticas de cuidado intergeracionais, suportadas por um conhecimento e comportamento cultural específico que garante a proteção das mulheres e seus filhos. As seguintes práticas de cuidado cultural destacam-se nesta síntese interpretativa: o jemené: rito que marca o início da mulher Embera Katio para a vida fértil; a alimentação: uma forma de preservar o bem-estar da mãe e do feto; a verticalização do parto e o uso de plantas medicinais durante o trabalho de parto; $o$ aconselhamento e acompanhamento da parteira tradicional durante a gravidez, parto e pós-parto e, por último, descanso e harmonia da mãe e do recém-nascido no período pós-parto.

Palavras-chave: Mulheres Indígenas, Grupos Étnicos, Práticas, Cuidado Cultural.

\section{INTRODUCCIÓN}

La cultura puede entenderse desde la perspectiva de Itcharth y Donati como un conjunto de patrones o modelos de comportamiento social presentes en una comunidad o grupo poblacional específico (1). La cultura encierra, entre otras prácticas, una serie de costumbres, códigos y normas que son reflejo de la dinámica social de un grupo (2). 
Ramos Lafont indica que en muchas comunidades indígenas existen prácticas de cuidado cultural, caracterizadas por mantener un conjunto de costumbres ancestrales que son empleadas para asegurar su supervivencia y conservación; estas prácticas culturales son transmitidas de generación en generación, especialmente por la mujer, quien es la protagonista de la conservación de su legado (3). Las prácticas de cuidado cultural en mujeres indígenas están estrechamente enlazadas con su cosmovisión (4). Comprender las prácticas de cuidado cultural en el continuo reproductivo de la mujer Embera se convertirá en una fuente valiosa de información para generar estrategias que permitan un acercamiento con la mujer durante este periodo.

Para los profesionales de enfermería contar con competencias de cuidado cultural resulta una tarea fundamental, toda vez que esta competencia le permitirá negociar, re-estructurar o mantener las prácticas de cuidado cultural con las comunidades o grupos especiales con los que interactúa (5).

En el departamento de Córdoba en Colombia existen diversos grupos étnicos: entre ellos los Embera Katio; de este grupo poblacional, las mujeres son vulnerables a la atención en salud porque muchas veces los profesionales de la salud desconocen sus prácticas culturales y la forma de concebir sus procesos reproductivos; es por ello que la presente investigación se plantea como pregunta de estudio ¿Cuáles son las prácticas de cuidado cultural en el continuo reproductivo de la mujer Embera Katio del Alto Sinú?

Geográficamente el departamento de Córdoba está localizado en la parte noroccidental de Colombia, sobre la extensa llanura del Caribe. Este departamento, está conformado políticamente por 30 municipios, divididos en cuatro subregiones que son: la subregión de la cuenca del río Sinú, la Sabana, la Cuenca del río San Jorge y la Zona Costanera (6). La población indígena del departamento se ubica principalmente en los municipios de Tuchín, San Andrés de Sotavento y Tierralta. El municipio de Tierralta es el escenario donde se realizó este estudio. Tierralta es considerado el municipio más extenso del departamento de Córdoba, conformado por 18 corregimientos, 234 veredas y 4 reasentamientos de la etnia indígena Embera Katio. Este municipio se encuentra en la zona de influencia de la cuenca alta del río Sinú, el cual en su recorrido es alimentado por los siguientes afluentes: río Verde, Esmeralda y Manso; históricamente estas tierras han sido utilizadas para la siembra de cultivos ilícitos y como corredor de grupos al margen de la ley, especialmente la zona sur y la zona montañosa del territorio (7).

Los Embera Katio representan el 2,7\% de indígenas en Colombia; el departamento de Córdoba es el segundo del país con la mayor concentración de población Embera, alojando alrededor de 5.132 personas de esta etnia, es decir el 13,4\% de la población Embera del país (8). Las comunidades Katio asentadas en el departamento de Córdoba están organizadas por familias extensas, lideradas por el hombre mayor (abuelo), quien representaba la autoridad, pero después de la Ley 89 de 1890, se instauró el Cabildo como forma de organizar la vida política de sus comunidades (9, 10). El Cabildo es una entidad pública especial, cuyos integrantes son miembros indígenas, elegidos y reconocidos por ésta, con organización sociopolítica tradicional y su función es representar legalmente a la comunidad, ejercer autoridad y realizar las actividades que le atribuyen las leyes. 


\section{METODOLOGÍA}

Se realizó una investigación cualitativa de tipo etnográfico-interpretativo; este enfoque busca describir, explicar e interpretar un fenómeno de estudio $(11,12)$. Esta investigación se materializó con las posturas planteadas por Guber, quien afirma que la etnografía se puede concebir como enfoque, como método y como texto (13). Para este estudio se escogió la etnoenfermería como método, porque el interés de los investigadores fue comprender las prácticas de cuidado cultural en el continuo reproductivo de la mujer Embera (14).

Se utilizó un muestreo intencional, haciendo referencia a los casos disponibles; bajo esta perspectiva se encontraron dos tipos de colaboradores en coherencia con lo descrito por Leininger: los colaboradores culturales generales y los colaboradores clave (15). La selección de los colaboradores estuvo a cargo de los investigadores, teniendo en cuenta los principios de pertinencia, adecuación, conveniencia, oportunidad y disponibilidad (16).

Los colaboradores participativos fueron los líderes comunitarios, mujeres adolescentes y adultas de la comunidad indígena de Tuis-Tuis del municipio de Tierralta Córdoba. Los colaboradores generales, fueron un líder adulto, un líder joven y el maestro de la comunidad indígena, con quienes inicialmente se realizó acercamiento para conversar sobre las prácticas de cuidado cultural en el continuo reproductivo de la mujer indígena de la comunidad Embera Katio del Alto Sinú. Estos colaboradores permitieron la inmersión al campo y aportaron información que facilitó el acercamiento con los colaboradores clave.

Con el ánimo de lograr la saturación de la información, se efectuó observación participante en cuatro ocasiones; en cada una de estas, se realizó una entrevista etnográfica a cada colaborador clave y después del análisis, se decidió hacer otra entrevista para colmar los vacíos existentes. Los colaboradores clave ( 8 mujeres indígenas Embera Katio en edad reproductiva, en gestación y posparto) quienes participaron libremente en la entrevista etnográfica.

En cuanto al proceso para la recolección de la información, ésta se dio en dos momentos en el curso de la investigación: la inserción y el trabajo de campo. La inserción de campo se refiere a las primeras observaciones, diarios de campo y formas de organizar la información obtenida. Se llevó a cabo con la visita de reconocimiento y socialización del proyecto de investigación. En estas visitas se hicieron encuentros con los líderes de la comunidad, a quienes se les compartió de manera informal el proyecto y de quienes se obtuvo información general de los indígenas y las indicaciones a seguir para obtener los permisos necesarios.

Posterior a la etapa de inserción se pasó al trabajo de campo donde se pudo asistir, observar, grabar y participar en reuniones con la comunidad, previo permiso y consentimiento informado de los colaboradores.

Para la obtención de datos se utilizó la observación participante, el diario de campo y la entrevista etnográfica. La observación participante se ejecutó en dos etapas, permitiendo el desarrollo de la investigación; la primera fue observar y no participar, observando sistemáticamente lo que acontecía en la comunidad sin intervenir con las informantes clave; este tipo de observación se 
realizó en la inserción de campo cuando se realizaban las visitas de reconocimiento y las reuniones de socialización del proyecto.

La segunda etapa corresponde al involucramiento versus separación, proceso en el cual los investigadores tuvieron contacto con los colaboradores clave y la observación se separa del involucramiento que los une (17). Todas las entrevistas de los encuentros fueron grabadas en audios y apoyadas con los diarios de campo y los registros de los investigadores.

La entrevista etnográfica es considerada una conversación dirigida a la comprensión de las perspectivas del investigador y de los investigados, con el fin de captar los puntos de vista de los colaboradores respecto al fenómeno que se estudia (18).

En este estudio el análisis de datos se realizó con base a la guía del facilitador del Sol Naciente propuesto por Leininger desde la etnoenfermería, como parte del método para proveer un análisis riguroso, profundo y sistemático de los datos cualitativos (19). Esta guía sirvió para comprender los registros de los diarios de campo y la descripción de las zonas que no habían sido totalmente exploradas; además fue un mapa cognitivo que permitió entender los principales componentes de la teoría, mientras se recogían los datos y se analizaban los resultados como se muestra en el gráfico 1. 
Gráfico 1. Facilitador del Sol Naciente representando las prácticas de cuidado cultural en el continuo reproductivo de la mujer Embera Katío del Alto Sinú
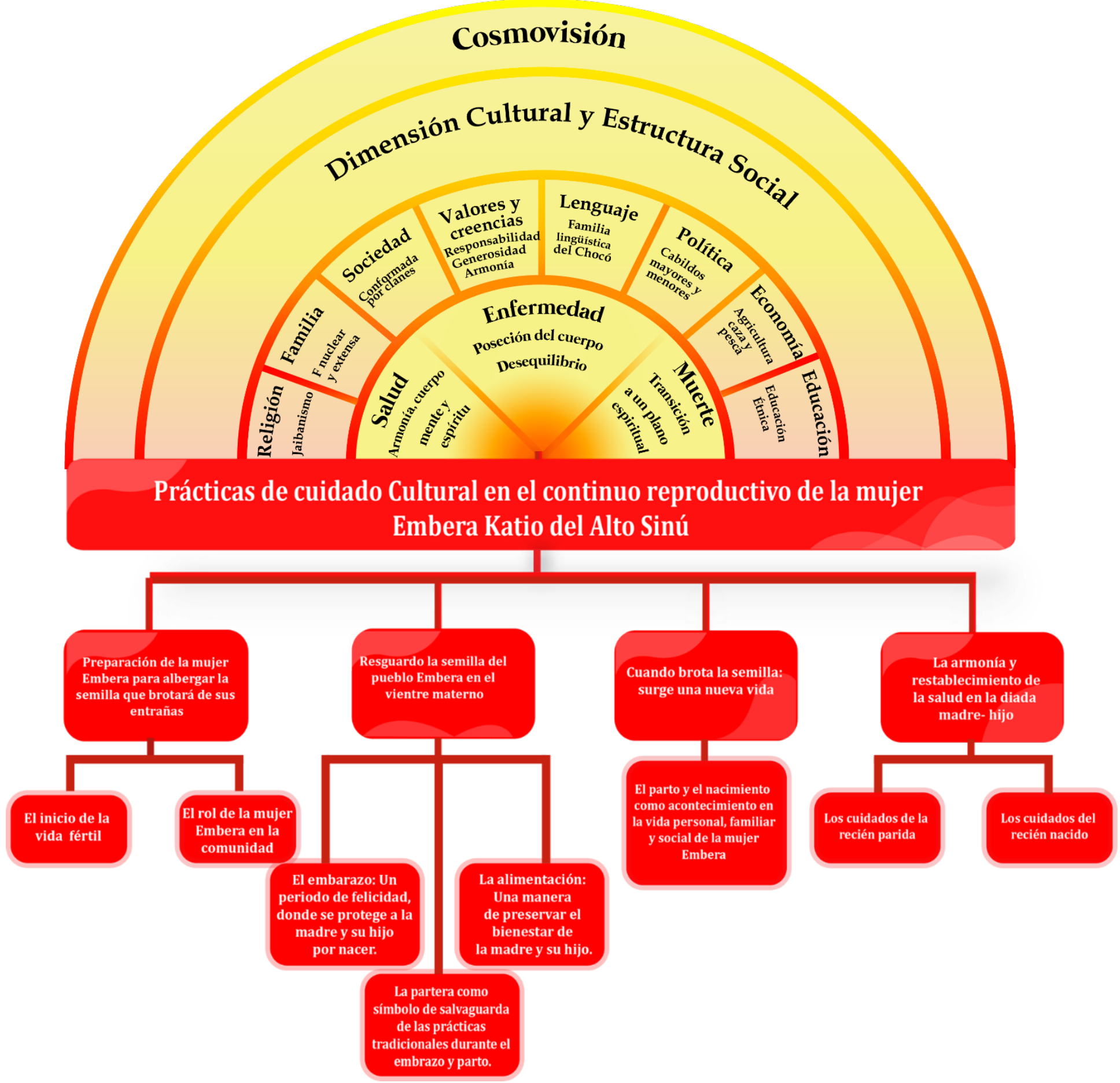
A partir del facilitador del Sol Naciente propuesto por Leininger, se realizó el análisis de los datos cualitativos, teniendo en cuenta las siguientes fases:

Gráfico 2: Diferentes fases del trabajo de investigación.

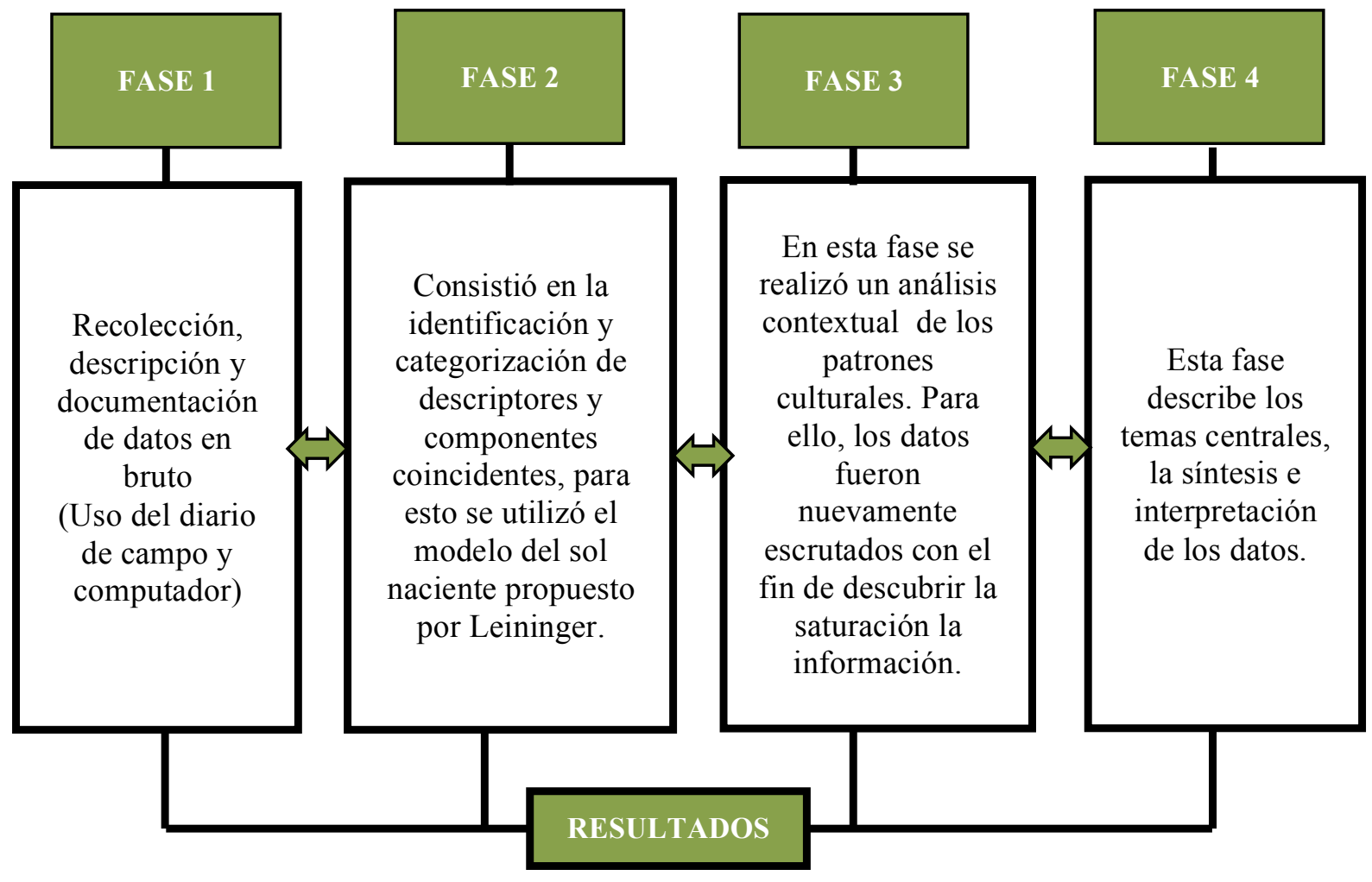

Fuente: Elaboración propia (2018)

\section{RESULTADOS}

Una vez transcritas las entrevistas, los investigadores realizaron una extensa revisión bibliográfica e iniciaron la identificación de fragmentos con sentido (descriptores) los cuales fueron codificados utilizando un término genérico, relacionado con las etapas del continuo reproductivo de la mujer Embera Katio, el cual fue traducido por un intérprete de la comunidad, como se ilustra en el siguiente ejemplo:

I2 (Yizaketoma) E4P5 "Ya uno sabe cuándo ya casi va pa parir!, uno busca la partera; si ella dice que está de cabeza, ya uno queda tranquila y ya uno sabe que va parir bien...

Donde:

I2: Informante 2 (Yizaketoma. Traduce a /Recién parida)

E4: (Entrevista 4).

P5: (Párrafo 5). 
Una vez depurada la información se conservaron los fragmentos significativos por cada colaborador clave; finalmente a través de mapas conceptuales, diagramas y dibujos se realizó un análisis comparativo, donde se asignaron códigos cuyo significado era similar y, se agruparon dando lugar a patrones, porque existían aspectos en común que los unía. Seguidamente, se volvieron a analizar los patrones buscando similitudes y diferencias, hasta agruparlos en cuatro temas principales que describen las prácticas de cuidado cultural en el continuo reproductivo de la mujer Embera Katio del Alto Sinú.

De acuerdo a la Teoría de la Universalidad y Diversidad de los Cuidados Culturales, el cuidado cultural debe ser visto como un elemento esencial para la salud humana, el bienestar y la supervivencia (20). De hecho, Leininger indica que el cuidado basado en la cultura es esencial para ayudar a las personas en la curación, recuperación, la discapacidad, enfrentamiento de la muerte (21).

A continuación, se presenta la síntesis interpretativa de cada una de las categorías temáticas que surgieron de esta investigación:

Tema 1. Preparación de la mujer Embera para albergar la semilla que brotará de sus entrañas.

La mujer Embera Katio en su propia cultura es vista como una persona importante por su capacidad de dar vida y asegurar la extensión de su etnia a través del tiempo; por esta razón, desde muy temprana edad son preparadas para para albergar la semilla que brotará de sus entrañas y asumir la responsabilidad de perpetuar la cultura Embera Katio.

Una subcategoría de análisis que se deriva del primer tema es El inicio de la vida fértil. El inicio de la vida fértil de la mujer Embera Katio lo marca el ritual del Jemené (término para referirse al ritual que realizan en la pubertad de la mujer después de la primera menstruación). Antes de llegar a esta etapa la mujer es educada para avisar a la madre la presencia del primer sangrado menstrual y dar inicio a los preparativos de la ceremonia. Este ritual se divide en tres momentos: el encierro, que tiene una duración variable y está sujeta a la finalización del ciclo menstrual; el baño en el río que se realiza con el fin de limpiar a la joven para fortalecer su cuerpo, y la fiesta, donde se integra la comunidad pidiendo a los espíritus protectores (jai) que acompañen a la joven en su vida adulta (22).

¡Una luna después, me hicieron una fiesta!... ¡me pintaron con jagua!... ¡Primero me encerraron en un cuarto donde sólo entraba mamá!..., ¡después me tiraron al río y en la fiesta me daban trago!... ;yo me emborraché!...

C1 E1P2 (awera/adolescente)

La segunda subcategoría identificada fue el Rol de la Mujer Embera en la comunidad. La mujer Embera Katio tiene funciones marcadas dentro de su comunidad que abarcan la preparación de alimentos, la fabricación de bebidas, el transporte de leña, la colecta de productos agrícolas para el hogar, el acarreo de agua y el cuidado de su esposo e hijos. El proceso de formación inicia desde muy temprana edad; los padres son los encargados de enseñar estas prácticas con el fin que en un 
futuro se puedan desenvolver como mujeres de bien, cuando decidan iniciar una familia. Un claro ejemplo de esta situación se evidencia con la siguiente narración:

¡Yo aquí estoy pa lavá, cociná, criar los hijos y darles consejos!...; ¡Pa criarlos bien!... ¡uno sale a buscá leña, a veces sale a buscá plátano, y está en la casa!..

C4E2P3 (yondrara/adulta)

Tema 2. Resguardando la semilla del pueblo Embera en el vientre materno.

El embarazo desde la cultura Embera Katio es considerando un acontecimiento social y familiar, enmarcado en la felicidad por la llegada de un nuevo ser. Este acontecimiento significa expansión de la familia, la cultura y la comunidad; por esta razón, en el embarazo se procura conservar la salud y bienestar de la madre y la semilla que se alberga en el vientre materno, mediante actividades que protejan la salud de la diada madre-hijo (23).

En este tema surgen tres subcategorías; la primera, El Embarazo: un periodo de felicidad, donde se protege a la madre y su hijo por nacer. La mayoría de las mujeres identifican su embarazo por síntomas como la amenorrea, las náuseas, el vómito, la pérdida de apetito y la debilidad. Para ellas, la identificación de signos y síntomas del embarazo hacen parte de un acontecimiento normal en su ciclo reproductivo.

Las embarazadas reconocen la importancia de sentirse apoyadas por la familia, especialmente por su madre, sus hermanas y cuñadas como se puede apreciar en la siguiente expresión:

¡A veces a uno lo cuida la mamá o están las cuñás!... ¡ya despué queda uno en casa cuidando bebé y marido de uno!.

C3E3P7 (biogo/embarazada)

Es importante mencionar que dentro de las creencias culturales se considera el embarazo como un acontecimiento natural en la vida de la mujer, donde ellas siguen sus creencias y prácticas tradicionales, además de los consejos que otorga su familia (24).

Las gestantes consideran que ciertos oficios del hogar conllevan a esfuerzos que pueden ser nocivos para la salud de ellas y de su hijo por nacer; algunos de ellos son: cargar objetos pesados, cargar agua en baldes, lavar ropa y cualquier otra actividad que le implique movimientos bruscos y cambios de equilibrio, por ser actividades que al efectuarlas originan en ellas una "mala fuerza" como se puede confirmar en la siguiente narración.

¡Cuando uno está embarazá tiene que hacer los oficios, pero no que sean pesados!... ¡una mala fuerza daña a bebé!..., ¡uno se cuida mucho de cargar leña, agacharse mal y todo eso!... C2 E4 P6 (biogo/ embarazada)

La segunda subcategoría del tema 2 se denomina La Alimentación: una manera de preservar el bienestar de la madre y su hijo por nacer. En la búsqueda de la protección de la salud materna, las gestantes consideran que cuidarse en la alimentación es una forma de proteger su propia salud y la de su hijo por nacer. La alimentación tiene una interpretación y un significado particular de 
beneficio, protección y seguridad, lo que hace que las mujeres gestantes lleven a cabo prácticas o formas para cuidar su alimentación desde sus propias creencias, valores personales y del núcleo familiar, que buscan preservar el bienestar de ambos.

¡Yo no como ahuyama, eso pone cabezón el bebé y despué atranca el parto!..., ¡tampoco papaya ni piña porque tienen granos y despué bebe sale asi!...

C3 E2 P5 (biogo/embarazada)

Alrededor de la alimentación existe también toda una serie de rituales encaminados a garantizar en la madre un buen parto, a partir del consumo de alimentos que dan fuerza, bebidas que calman los dolores de la contracción y facilitan la dilatación y el borramiento en el momento del parto.

La tercera subcategoría es La partera como símbolo de salvaguarda de las prácticas tradicionales durante el embarazo y parto. La figura de la partera en la comunidad Embera Katio se reconoce como una mujer sabia porque tiene un conocimiento cultural y simbólico especial para brindar cuidado a las mujeres durante el embarazo, la asistencia del parto, el postparto y el cuidado del recién nacido.

¡Ella da consejo!..., ¡ayuda a las primerizas!..., ¡si uno quiere sabé si es macho o hembra el bebé, la partera dice!... jexprime la teta, si la leche se corre en la totuma es hembra!.., ipero si cae concentrá es macho!...

¡Ella me acompañó con mi primer hijo!..., ;los otros yo los parí solo con mi mamá!...

C5 E3 P2 (yondrara/adulta)

En esta comunidad, una de las maneras empleadas para cuidarse durante el embarazo y garantizar la protección de su hijo por nacer es la visita a la partera, en especial cuando las gestantes son primerizas.

¡Ya uno sabe cuándo ya casi va pa parir!..., !uno busca la partera!...; ;si ella dice que está de cabeza, ya uno queda tranquila y ya uno sabe que va parir bien!...

C6 E1 P8 (Yizaketoma/recién parida)

Las parteras indican a las mujeres cómo va el embarazo, si el bebé se encuentra bien acomodado, qué sexo es y si todo se encuentra bien para el parto; también les soban la barriga cuando sienten algún dolor o cuando sienten que el bebé se les encaja o se desacomoda.

¡Yo sobo barriga para que bebé quede con cabeza pa abajo!...

C7 E4 P5 (partera)

En la práctica de "sobar" la partera empuja la barriga de izquierda a derecha y de atrás hacia adelante, lo más fuerte que la gestante resista con el fin de desplazar al bebé hacia abajo.

Tema 3. Cuando brota la Semilla: surge una nueva vida.

De este tema surge la siguiente subcategoría, El parto y el nacimiento como acontecimiento en la vida personal, familiar y social de la mujer Embera Katio. De manera comprometida la familia se interesa en forma particular por el bienestar físico, emocional y espiritual de la parturienta, al 
orientar acciones de cuidado para proporcionar a la mujer y al recién nacido condiciones de seguridad durante el trabajo de parto y parto.

¡Mi familia siempre me apoya!..., ;un hijo siempre es bienvenido!... iyo tengo ocho hijos, y los últimos los parí sola en la casa!...

C5 E2 P6 (yondrara/adulta)

¡Yo me voy alistando pal parto!... ;Yo tengo los trapos listos para que bebé caiga!..., ¡cuando voy a parir tengo que agacharme y abrir las piernas agarrando un palo y a las tres jalás sale bebé!...

C6 E2 P9 (Yizaketoma/recién parida)

¡Yo amarro con hilo el ombligo, despué separo sangre y se mocha la tripa de ombligo, con cuchilla nueva!...

C7 E3 P3 (partera)

¡Despué de parir me baño con agua de chivini y parará!...; ¡bebé también, eso pa que yo no enferme y bebé tampoco!....

C6 E3 P4 (Yizaketoma/recién parida)

Los temas descritos anteriormente giran alrededor del cuidado del embarazo como una manera de prepararse para el parto; con este propósito se ha observado y documentado cómo las mujeres empiezan a alistarse para el nacimiento de su hijo, desde el momento que se enteraron de su estado de gestación, evidenciado por un cambio en su estilo de vida y en la adopción de prácticas de cuidado, afianzadas en sus creencias y valores personales e influenciadas por las experiencias de otras mujeres.

Tema 4. La armonía y restablecimiento de la salud en la diada madre-hijo.

Como subcategorías de este tema surgieron:

Los cuidados de la recién parida. Dentro del marco de conceptos y prácticas de cuidado que realiza la recién parida, está el concepto de "evitar recoger el frio" esta situación las lleva a una serie de prácticas que le permite mantener el cuerpo en equilibrio entre el frío y el calor; estos conocimientos tradicionales les garantiza estar bien después de parir.

¡Uno pare encerrao, no puede coger frío!..., jeso hace daño!... uff... hace mucho daño! ... ¡A uno puede entrarle frío y la barriga le duele después del parto!..., ipor eso uno se cuida mucho!...

C8 E4 P5 (Yizaketoma/recién parida)

El frío se convierte para ellas en una sensación "incómoda", porque dependiendo del lugar por donde entra el frío, se comienza a producir alteraciones en el cuerpo de la mujer, con consecuencias dañinas que no sólo afectan a la madre, sino que también afectan al bebé.

La alimentación y el reposo también juegan un papel importante en este periodo; para la cultura Embera Katio, los alimentos de elección en el posparto son las aves de corral y bebidas azucaradas. 
Asimismo, procuran limitar sus actividades rutinarias hasta sentir mejoría para reiniciar sus actividades en el hogar.

¡Yo comía gallina, chocolate y panela cuando paría!... ;Yo comía todo eso para que se reponiera la barriga!..., ;dicen que la sangre se queda en la barriga, entonces la panela ayuda a que eso baje y cuando se toma caliente el agua de panela es más rápido que baje esa sangre y así no tener apuros y dolores!... C4 E3 P7 (yondrara/adulta)

¡Uno debe está quietecita, quietecita, mientras que pase dieta!... ¡No puede lavá, no puede hacé na de oficio durante una luna, ya despué sí, pero na pesado!. C8 E4 P6 (Yizaketoma/recién parida)

¡Pá aflojá la teta, uno se saca la leche y le echa sal!..., ;despué se soba la teta o se hecha agua tibia! C5 E3 P5 (yondrara/adulta)

Los cuidados del recién nacido. En el periodo posterior al nacimiento, el recién nacido recibe una serie de cuidados por parte de su madre con el fin de protegerlo y evitar enfermedades; estos cuidados se traducen en una alimentación exclusiva con leche materna durante los primeros tres meses y luego van añadiendo otros alimentos, también incluye los cuidados del ombligo con talco y agua hervida hasta que este se caiga, al igual que la protección del recién nacido con baños de plantas y jagua ${ }^{1}$, entre otras plantas medicinales.

¡Cuando bebé nace lo baño con agua tibia y jabón!..., ¡después de una luna de nacido, baño a bebé con agua de hojas de matarraton, hojas de limón y hojas de chiviní para que no se enferme y tenga fortaleza!... C8 E2 P1 (Yizaketoma/recién parida)

¡Despué que bebé nace, doy solo teta!..., ; a los tres meses se caza un pajuil, se saca la pechuga, se muele y le echo condimento, se hace una especie de albóndiga y se llama a una persona mayor para que le dé su primera comida de sal! C4E3P7 (yondrara/adulta)

Una forma de proteger la piel del recién nacido justo después del parto, es untarle la placenta en todo el cuerpo como símbolo de belleza y a la vez de protección.

¡Yo unto a bebé la placenta, para evitar salpullido!..., ¡también pongo un juuajú ${ }^{2}$ en la manito para protegerlo de los espíritus malos, a los días de nacido se dibujan unos kipará ${ }^{3}$ en todo el cuerpo pá protegerlo de la maldad! C5 E4 P6 (yondrara/adulta

Finalmente, en el gráfico 3, se presentan las prácticas de cuidado cultural identificadas en el continuo reproductivo de la mujer Embera Katio del Alto Sinú, entendiéndose como un proceso armónico que tiene un punto de inicio y un punto de finalización que se repite constantemente.

\footnotetext{
${ }^{1}$ Fruto sagrado del cual se extrae su tintura para curar enfermedades y embellecer el cuerpo mediante tatuajes.

${ }^{2}$ Hace referencia a una especie de amuleto utilizada por la etnia Embera para la protección de los recién nacidos.

${ }^{3}$ Pintura corporal Embera, para protección de espíritus malignos (jai) y de las enfermedades, utilizada además para dar fortaleza a los bebes.
} 
Gráfico 3. Prácticas de cuidado cultural identificadas en el continuo reproductivo de la mujer Embera Katio del Alto Sinú

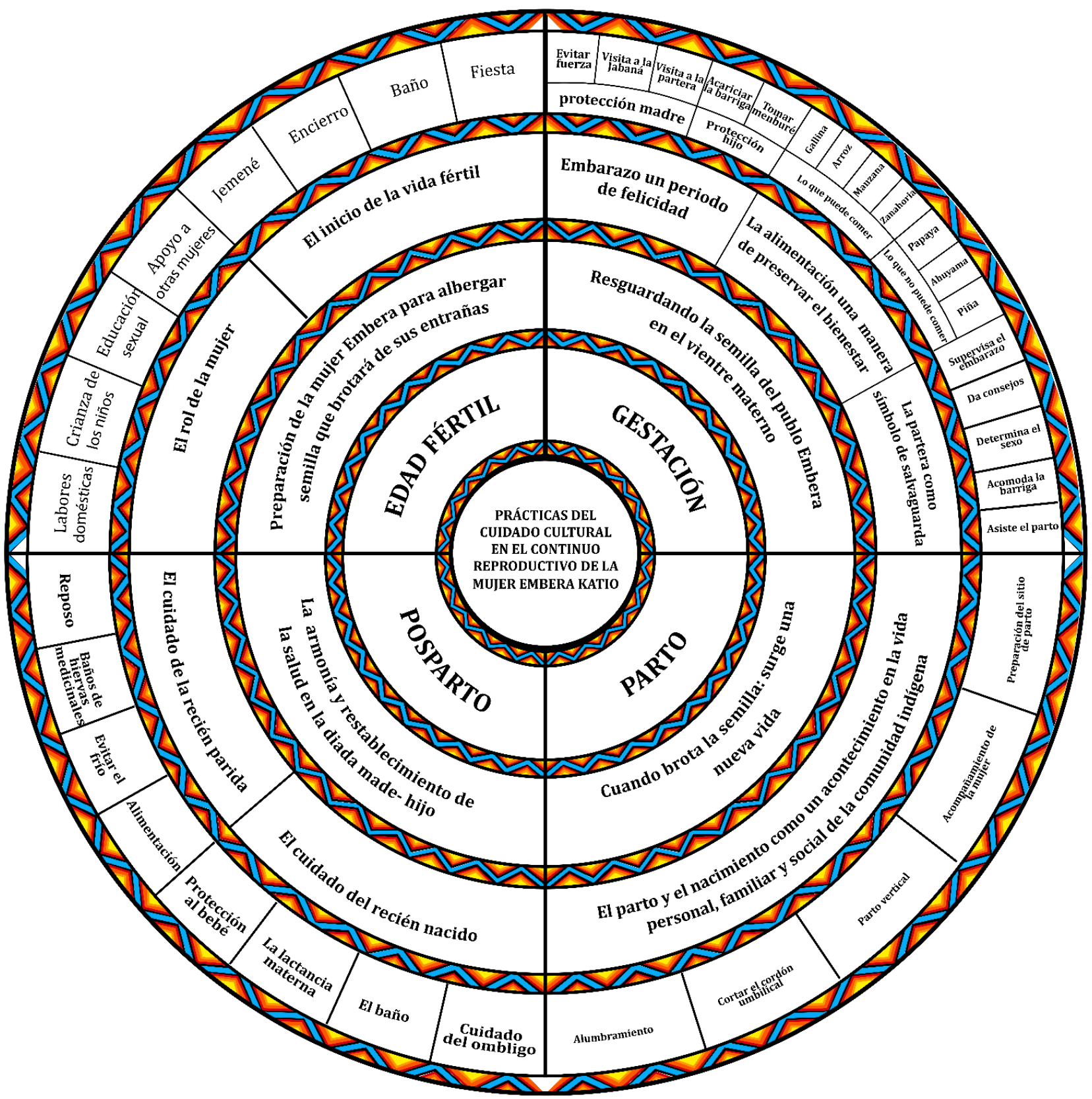

\section{DISCUSIÓN}

Identificar las prácticas de cuidado cultural en el continuo reproductivo de la mujer Embera Katio del alto Sinú permitió conocer diversas tradiciones culturales alrededor del ciclo reproductivo de la mujer, orientadas a su cuidado y al de su hijo por nacer. En esta investigación surgieron cuatro temas o categorías de análisis con sus respectivas prácticas de cuidados culturales. 
Existen escasos registros sobre las prácticas de cuidado cultural en el continuo reproductivo de la mujer Embera Katio del Alto Sinú, debido a que los constructos teóricos de estas prácticas culturales sólo son trasmitidos por tradición oral. La discusión que se presenta a continuación toma como referencia un grupo de estudios de otras etnias que documentan las prácticas de cuidado cultural en el continuo reproductivo de la mujer.

Los Embera Katio brindan educación a la mujer desde muy temprana edad para que ésta asuma el rol que le corresponde dentro de la comunidad. La vida fértil de una mujer Embera Katio inicia con la llegada de la primera menstruación; este acontecimiento está acompañado de rituales que preparan a la mujer para dotarla de conocimientos y fortaleza, indicando al resto de la comunidad que ya se encuentra apta para la procreación y conformación de una familia.

Una vez constituida la nueva familia, la mujer Embera Katio del Alto Sinú empieza su ciclo reproductivo, dando inicio a la extensión de la familia y repoblación de su etnia. El embarazo es considerado un periodo de felicidad donde la mujer se protege a sí misma y protege al hijo por nacer; de esta manera se despliegan una serie de prácticas de cuidado durante el embarazo, que son transmitidas ancestralmente de una mujer a otra, de generación en generación y están sujetas a las costumbres y las creencias propias de su cultura.

La investigación realizada por Hernández en 2012 evidencia que los conocimientos y las prácticas de cuidado cultural en las gestantes, obedecen a la información que han recibido de sus progenitoras, abuelas y bisabuelas, constituyéndose así en normas culturales que se transmiten de generación en generación (25). De la misma manera, el estudio realizado por Ramos Lafont evidencia que durante el embarazo las indígenas Zenúes tienen un fuerte arraigo a sus tradiciones y costumbres culturales, situación que no difiere de los hallazgos reportados en esta investigación (3).

La supervisión del embarazo en la mujer Embera Katio del Alto Sinú la brinda principalmente la partera, quien acompaña y orienta a la mujer sobre los cuidados que debe tener durante esta etapa. La partera ocupa un lugar privilegiado en la comunidad, por ser la figura que representa el conocimiento tradicional y el saber empírico de los procesos reproductivos de la mujer; generalmente una partera es solicitada para brindar consejos relacionados con el embarazo, parto y posparto, revisar la posición del bebé y en caso de ser necesario acomodarlo; también puede asistir el parto de la mujer primeriza o los partos difíciles, como se evidencia en la investigación realizada por Muñoz en las comunidades del Cauca (26).

Siguiendo los planteamientos anteriormente descritos, Argote y Vásquez sostienen que la maternidad se ve revestida de creencias, mitos y tabúes que muchas veces se representan en la figura de la partera o comadrona, quienes velan por la salud y el bienestar de las mujeres durante la gestación, el parto y el posparto y motivan tanto a las madres como a sus familiares a que se unan al proceso de cuidado (27).

Desde la cosmovisión Embera Katio, los alimentos que se consumen durante la gestación pueden determinar la existencia de una complicación durante el embarazo y el parto, o afectar la condición 
física del niño. La investigación realizada por Medina identificó que las gestantes Wounaan no ingieren algunos alimentos para evitar dificultades durante el parto, asignando también características físicas a la estructura ósea y cefálica del recién nacido por haber consumido ciertos alimentos (28).

En esta investigación se encontró que la alimentación es una práctica importante para conservar la salud de la madre y el bienestar de su hijo por nacer; en este sentido, la mujer Embera Katio, tiene un conjunto de prácticas alimenticias relacionadas con lo que pueden y no pueden comer y éstas se encuentran determinadas por las tradiciones de su cultura; esta misma situación se evidencia en algunas comunidades indígenas del Perú como lo reporta la investigación realizada por Chávez (29).

Las costumbres y creencias de una cultura determinan la seguridad del parto y la preparación de la mujer para un nacimiento sin complicaciones; por otro lado, la gestación se reconoce como un proceso natural de la mujer que se encuentra ligado a diversas costumbres y creencias que se transmiten de generación en generación y se conservan a través de la experiencia de un grupo social $\mathrm{y}$ de un contexto familiar, que tienen como finalidad conservar la salud y el bienestar de la madre y su hijo por nacer $(30,31)$.

Otro aspecto de importancia para la cultura Embera Katio del Alto Sinú hace referencia al acompañamiento de la mujer durante el proceso de parto. La mujer que da a luz es el centro de la atención y unión familiar. La mujer Embera pare en su propio hogar, porque éste le brinda la comodidad y calidez necesaria para estar tranquila. El parto se realiza con el apoyo de la partera de la comunidad cuando las mujeres no son experimentadas; la mayoría de las veces se realiza con el acompañamiento de un familiar con experiencia en la atención del parto como madres, abuelas, hermanas o cuñadas y en algunos casos su propio esposo.

El cuidado de la mujer Embera Katio del Alto Sinú en el posparto está a cargo de ella misma, de su madre o las mujeres más cercanas a ella; en el último de los casos el esposo. La persona a cargo del cuidado de la recién parida asume las tareas que anteriormente realizaba la mujer en la cotidianidad de su hogar, teniendo en cuenta que ésta debe abstenerse de hacer esfuerzo físico o cargar objetos pesados como lo plantea Ortega en su investigación, donde las mujeres indígenas después del parto no pueden cargar cosas pesadas, no pueden comer ciertos alimentos, evitan ponerse en contacto con ciertas personas o visitar determinados lugares (32).

El periodo de posparto inicia con la recuperación de la madre y el cuidado del recién nacido; en la comunidad Embera Katio del Alto Sinú la recuperación de la madre depende principalmente de la alimentación, la cual es a base de aves de corral y bebidas azucaradas.

En lo que se refiere al cuidado del recién nacido, se resaltan algunas prácticas de cuidado cultural, especialmente el cuidado del ónfalo, la protección del recién nacido de espíritus que representen una amenaza para su bienestar, los baños con plantas medicinales, la lactancia materna exclusiva los primeros tres meses y la protección de su cuerpo con Kipará o jagua para fortalecer su carácter y brindar protección a su piel contra las picaduras de zancudo. 


\section{Reflexión de la síntesis interpretativa de las prácticas de cuidado cultural en el continuo reproductivo de la mujer Embera Katio del Alto Sinú}

Enfermería debe acceder al mundo de la mujer Embera Katio, tomar sus puntos de vista, sus conocimientos y prácticas $\mathrm{y}$, sumados a los conocimientos profesionales, preservar y/o mantener, negociar y reestructurar patrones de cuidado cultural, para brindar un cuidado seguro, benéfico y culturalmente congruente, orientado a la protección de la madre y su hijo por nacer.

Desde la perspectiva del cuidado transcultural, el profesional de enfermería podrá brindar un cuidado humanizado, coherente y congruente con las creencias, valores y prácticas de cuidado a la mujer Embera Katio en el continuo de su proceso reproductivo, disminuyendo los conflictos y choques transculturales que hay entre el conocimiento disciplinar de enfermería y su propia cultura.

\section{CONCLUSIONES}

El continuo reproductivo de la mujer Embera Katio del Alto Sinú está representado por un conjunto de prácticas de cuidado de orden intergeneracional soportadas en un conocimiento $y$ comportamiento cultural específico, que garantiza la protección de la mujer y su descendencia.

Entre las prácticas de cuidado cultural que se destacan de esta síntesis interpretativa se encuentran: el Jemené: Rito que marca el inicio de la mujer Embera Katio a la vida fértil; la alimentación: una manera de preservar el bienestar de la madre y su hijo por nacer; la Verticalización del parto y el uso de plantas medicinales durante el trabajo de parto; los consejos y el acompañamiento de la partera tradicional durante el embarazo, parto y posparto; el reposo y la armonía de la madre y el recién nacido en el posparto.

Se destaca el papel de la partera como cuidadora y como pieza clave para salvaguardar las creencias y prácticas de cuidado cultural en la comunidad Embera Katio del Alto Sinú, por la experiencia y el conocimiento que poseen para dar consejos a las mujeres en el continuo de su ciclo reproductivo.

\section{REFERENCIAS BIBLIOGRÁFICAS}

1. Itcharth, Laura y Donati, Juan. Prácticas culturales. En: Universidad nacional Arturo Jauretche. Chile. 2014. p.16.

2. Laza Vásquez, Celmira y cárdenas, Fernando José. Una mirada al cuidado en la gestación desde la enfermería transcultural. En: Revista Cubana de Enfermería. 2008, vol. 24, no. 3-4, p. 5. [En Línea]. [12 de marzo de 2012]. Disponible en: www.scielo.sld.enf09308.pdf )

3. Ramos Lafont, Claudia P. Prácticas culturales de cuidado de gestantes indígenas que viven en el resguardo Sinú. Bogotá, 2011.p. 11.

4. Bula, Javier y Galarza, Keiner. Mortalidad materna en la gestante wayúu de Uribía, departamento de la Guajira, Colombia. Estudio descriptivo año 2016. En: ENFERMERÍA: CUIDADOS HUMANIZADOS. Vol. 6, no. 1, p. 46-53. 
5. Ulloa Sabogal. Cuidado cultural en mujeres con embarazo fisiológico: una meta-etnografía. Universidad Nacional de Colombia Facultad de Enfermería Bogotá, Colombia. 2014

6. Secretaria Departamental de Salud. Departamento de Córdoba. Análisis de La Situación de Salud del Departamento de Córdoba ASIS 2013.

7. Secretaria Departamental de salud. Plan de Desarrollo departamental Córdoba, 2016-2019 www.cordoba.gov.co/descargas/Unidos porCordoba.pdf

8. Informe Final Mira Comunidades Indígenas Embera Katio del Alto Sinú. Disponible EN: www.humanitarianresponse.info/es/.

9. REPÚBLICA DE COLOMBIA. MINISTERIO DEL INTERIOR. Ley 89 de 1890. Disponible en: www.icbf.gov.co.

10. COMISIÓN INTERAMERICANA DE DERECHOS HUMANOS. Capitulo XI los derechos de los indígenas en Colombia. En línea: www.cidh.org/countryrep/Colombia93sp/cap.11.htm.

11. Sandoval Casilimas, Carlos. Investigación Cualitativa; Programa de especialización en teoría, métodos y técnicas de investigación social; Instituto Colombiano para el Fomento de la Educación Superior (ICFES); Bogotá, Colombia. 1996. p 11.

12. Hernández, Sampieri Roberto; Fernández, Collado Carlos; Baptista, Lucio Pilar. Metodología de la investigación, Cuarta edición, Ed. Mc Graw Hill. El proceso de la investigación cualitativa, Tercera parte. 2010. Cap.14, p. 583.

13. Guber, R. (2007). La etnografía. Método, campo y reflexividad. Bogotá: Norma. Hernández, Luz. La gestación: proceso de preparación de la mujer para el nacimiento de su hijo (a). Avances en Enfermería. [Revista en Internet] 2008 [Acceso 12 de Septiembre de 2018] 26(1): 97-102. Disponible en: www.revistas.unal.edu.co/index.php/avenferm/article/view/12889.

14. Leininger Madeleine, Teoría de la universalidad y diversidad del cuidado cultural y evolución del método de la etnoenfermería cap. 1; p.1.2006.

15. Sandoval Casilimas, Carlos. Investigación Cualitativa; Programa de especialización en teoría, métodos y técnicas de investigación social; Instituto Colombiano para el Fomento de la Educación Superior (ICFES); Bogotá, Colombia. 1996. p 11.

16. Guber, R. (2007). La etnografía. Método, campo y reflexividad. Bogotá: Norma.

17. Leininger Madeleine, Teoría de la universalidad y diversidad del cuidado cultural y evolución del método de la etnoenfermería cap. 1; p.1.2006 
18. Castillo, Edelmira y Vásquez, Martha Lucia. El rigor metodológico en la investigación cualitativa; Colombia Médica, vol.34, no. 3, 2003

19. Polit, D., \& Beck, C. (2011). Nursing Research: Generating and Assessing Evidence for Nursing Practice. Lippincott Williams and Wilkins.

20. Leininger Madeleine, Teoría de la universalidad y diversidad del cuidado cultural y evolución del método de la etnoenfermería cap. 1; p.1.2006

21. Leininger, Madeleine y McFarland, Marilyn. Culture Care Diversity and Universality. A Worldwide Nursing Theory. Chapter, 1. In: Culture Care Diversity and Universality Theory and Evolution of the Ethnonursing Method, Op. cit., p.4.

22. Cosmogonía: El Universo Embera y el Jaibanismo. [En línea] www.pueblosoriginarios.com/sur/caribe/embera/jaibanismo.html.

23. Los Embera Katio: Una Cultura Por Conocer, embera katio.2012. p.2. [En línea]: www.losemberakatio.com.co/2012/05/embera-katio.html.

24. Estudio etnosocial Embera-alto Sinú. En: Banco de la república, centro de documentación. Barranquilla. 1991. Tomo 2.p.111

25. Hernández, Luz. La gestación: proceso de preparación de la mujer para el nacimiento de su hijo (a). Avances en Enfermería. [revista en Internet] 2008 [Acceso 12 de septiembre de 2018] 26(1): 97-102. Disponible en: www.revistas.unal.edu.co/index.php/avenferm/article/view/12889.

26. Muñoz, Sandra, et al. Interculturalidad y percepciones en salud materno-perinatal, Toribio Cauca 2008-2009. Rev. Univ. Ind. Santander. Salud [revista en Internet]. 2012 abril [Acceso 19 de junio de 2013]; 44 (1): 39-44. Facultad Nacional de Salud Pública. Disponible en: www.revistas.uis.edu.co/index.php/revistasaluduis/article/view/2738

27. Argote, Luz Ángela, La donación hace la diferencia en el cuidado de padres e hijos, Fam, Saúde Desenv,Curitiba,v4,n1,p.7-15,jan/jun.2002

28. Medina, Armando y Mayca, Julio. Creencias y costumbres relacionadas con el embarazo, parto y puerperio en comunidades nativas Awajun y Wampis. Rev. Perú. Med. Exp. Salud Pública. Disponible en: www.scielo.org.pe/pdf/rins/v23n1/a04v23n1.pdf.

29. Chavez, Rocio, et al. Rescatando el autocuidado de la salud durante el embarazo, el parto y al recién nacido: representaciones sociales de mujeres de una comunidad nativa en Perú. Texto contexto-enferm. [Online].2007, vol.16, N.4, pp.680-687.ISSN01040707. www.scielo.br/scielo.php.es

30. García, Clotilde y de la cruz, Sabina. La salud perinatal de la mujer en una comunidad indígena. En: CIENCIA ERGO SUM. México, 2008. Vol.15, No. 2. p.151 
31. Medina, Armando y Mayca, Julio. Creencias y costumbres relacionadas con el embarazo, parto y puerperio en comunidades nativas Awajun y Wampis. Rev. Perú. Med. Exp. Salud Pública. Disponible en: http://www.scielo.org.pe/pdf/rins/v23n1/a04v23n1.pdf.

32. Ortega J. Géneros y generaciones: conducta reproductiva de los Mayas de Yucatán, México. Salud Colectiva [revista en Internet] 2006 [Acceso 10 de septiembre de 2018] 2(1): 75-89, 2006. Disponible en: www.scielo.org.ar/scielo.php. 